\title{
Endogenous vs. Exogenous Regulations in the Commons
}

October 28, 2015

\begin{abstract}
It is widely believed that there is strong experimental evidence to support the idea that exogenously imposed regulations crowd out the intrinsic motivations of common pool resource (CPR) users to refrain from over-harvesting. We introduce a novel experimental design that attempts to disentangle potential confounds in previous experiments. A key feature of our experimental design is to have the exact same regulations chosen endogenously as those that are imposed exogenously. When we compare the same regulations chosen endogenously to those externally imposed, we observe no differences in extraction levels among CPR users in a laboratory experiment. We also observe no differences between weak external regulations and no regulations, after controlling for a potential confound. However, when we add communication to our endogenous treatment, we observe significant behavioral differences between endogenous regulations with communication and exogenous regulations without communication. Our results suggest that externally imposed regulations do not crowd out intrinsic motivations in the lab and they confirm that communication facilitates cooperation to reduce extraction.
\end{abstract}

Keywords: Resource Management; Endogenous Rule-Making; External Regulations; Crowding Out

JEL Classification: C14, C7, C72, C91, Q2 


\section{Introduction}

One of the eight principles for successful common pool resource (CPR) management identified by Ostrom (1992) is that resource users affected by regulations should be included in the group that can modify these regulations. Case studies from the field suggest that self-organized systems of CPR management are successful when resource users take part in the decision-making process and management often fails when it is exogenously imposed "top-down" on resource users (Ostrom 1992). In her Nobel Prize address, Ostrom cited the experimental work of Cardenas et al. (2000) as evidence that externally imposed regulations can crowd out the intrinsic motivations of resource users to restrain their extraction. ${ }^{1}$ Understanding if, how, and why external regulations crowd out intrinsic incentives to conserve natural resources is of tantamount importance. If external regulations do crowd out internal incentives to protect the commons, then many environmental policies may actually be doing more harm than good.

This paper attempts to address some of the confusion and debate in the literature on endogenous vs. exogenous regulations in the commons with a novel experimental design. In a key departure from earlier work, our experimental design allows for the exact same regulations to be introduced endogenously or imposed exogenously. This addresses an important confound in the existing literature. We also introduce treatments that allow us to separately disentangle the effects of endogenous rule-making, between-subject communication, and strategic learning. Isolating these separate effects also allows us to identify potential confounds.

\footnotetext{
${ }^{1}$ Ostrom, like many scholars who cite Cardenas et al. (2000), misinterpreted slightly how Cardenas et al. (2000) reached their conclusion. She states that "subjects [in the regulation treatment] increased their withdrawal levels when compared to the outcomes obtained when face-to-face communication was allowed and no rule was imposed" (Ostrom 2009). However, their crowding out effect comes from a within-subjects analysis of their regulation treatment, not a between-subjects analysis between their regulation and communication treatments. That is, within their regulation treatment, Cardenas et al. (2000) observe higher individual extraction when an external regulation is weakly imposed compared to when no regulation is imposed.
} 
All of our results are remarkably consistent with each other. In our experimental context, we find no evidence that exogenously imposing regulations crowds out motivations to refrain from extraction. We show clearly that communication and strategic learning matter. If previous work has sometimes confounded communication with endogenous regulation and confounded strategic learning with exogenous regulation, then it is not surprising that existing results appear to contradict each other. Our aim is to use a simple experimental design to clear up some of this confusion. We start by reviewing the literature on external regulation and intrinsic motivation in Section 2, before giving a detailed description of our experimental design in Section 3. Section 4 summarizes our results and Section 5 concludes.

\section{Literature Review}

In her survey of economic experiments on common pool resources, Ostrom (2000) highlights the importance of endogenous rule making, where common pool resource users create their own rules, giving them a sense of accountability in the management of the resource. Many field examples of successful CPR management involve resource users in the rule-making process. In Torbel, Switzerland, a small village is able to manage communal lands in high mountain meadows and forests by enforcing rules that are voted on by all citizens in the village (Ostrom 1990). The Zanjera irrigation communities in the Philippines successfully irrigated their lands through a system that was devised and chosen by the farmers themselves in contrast to the failure of exogenously imposed irrigation systems in the Kirindi Oya project in Sri Lanka (Ostrom 1990, 1992). However, one of the difficulties with drawing causal inferences from case studies in the field is that many of these endogenously chosen systems may have worked because of the incentives they created, not necessarily because they were self-chosen.

Complementary to the documentation of a correlation between endogenous rule-making 
and successful CPR management is the idea that the alternative (externally imposed regulations) can actually crowd out intrinsic incentives to conserve natural resources. A vast literature (particularly in Psychology) has examined how imposing well-intentioned rules could crowd out an individual's intrinsic motivation, sometimes leading to worse results than if the intervention did not exist in the first place. ${ }^{2}$ A classic study on crowding out effects is Titmuss (1970), who studied the effects of monetary compensation on blood donation. Titmuss found that when individuals were monetarily compensated for donating blood, blood donation decreased. Similar crowding-out effects have been found among image-conscious volunteer firefighters (Carpenter and Myers 2010), parents who put their children in daycare centers (Gneezy and Rustichini 2000a), and IQ exam takers and volunteer donation collectors (Gneezy and Rustichini 2000b). ${ }^{3}$

Examples of the crowding out hypothesis in the environmental domain have been examined by Frey and Oberholzer-Gee (1997) and Kunreuther and Easterling (1990). Both papers study unwanted but necessary projects (such as hazardous waste facilities) and how monetary compensation crowded out an individual's sense of civic duty to accept the projects. Frey and Oberholzer-Gee (1997) find that "when public spirit prevails, using price incentives to muster support for the construction of a socially desirable, but locally unwanted, facility comes at a higher price than suggested by standard economic theory because these incentives tend to crowd out civic duty" (p. 753). Kunreuther and Easterling (1990) find that when the risk fell into an admissible range, individuals refused any form of monetary compensation.

Within the realm of CPRs, the most influential paper on crowding out effects is by Car-

\footnotetext{
${ }^{2}$ See, for example, the survey paper by Frey and Oberholzer-Gee (1997) and the meta-analysis by Deci et al. (1999).

${ }^{3}$ In a similar vein, Charness (2000) found that experimental subjects assigned as employees worked harder when their wage was determined randomly by a bingo cage than when it was determined by a neutral thirdparty individual: the responsibility that employees felt to work hard was "alleviated" when the wage was determined by another human being. Although an external intervention did not exist, it could be said that the employees' sense of responsibility was crowded out by the existence of an external third party in charge of determining wages.
} 
denas et al. (2000), who run a CPR lab experiment with and without regulations. ${ }^{4}$ In their experiment, groups of 8 foresters in rural Colombia played between 8 and 11 rounds of a CPR game without regulations and then played between 9 and 12 rounds of the same game with weakly enforced regulations. Their results show that resource extraction at the end of the second stage was higher than resource extraction at the end of the first stage, leading them to interpret their results as evidence that "regulation appeared to crowd out other-regarding behavior" (p.1719). However, in a different paper, using a similar experimental design in the same field setting, Cardenas (2004) no longer finds the same result, as externally imposed but weakly enforced regulations and communication both encourage lower extraction from CPR users. ${ }^{5}$ Other influential work on endogenous regulations in CPR experiments includes Bischoff (2007) and Vyrastekova and Soest (2003). Bischoff (2007) compares a CPR game with communication to a CPR game with an opportunity to endogenously change the regulations. The regulations are not the same across treatments but Bischoff (2007) concludes that endogenously-chosen strong regulation performs worse than weak regulation with communication. Vyrastekova and Soest (2003) explore whether allowing resource users to vote on who keeps the fines generated from enforcement enhances the efficiency of resource use. They find that harvesters infrequently voted to allow the enforcer to keep the fines generated, leading to inefficient outcomes.

Given the importance of Cardenas et al. (2000), it is useful to highlight the key differences

\footnotetext{
${ }^{4} \mathrm{~A}$ key difference between Cardenas et al. (2000) and the experiment presented in this paper is that Cardenas et al. (2000) is a lab experiment conducted in the field, whereas our study is a lab experiment conducted in the lab. See Anderson et al. (2013) and references therein for an important discussion of differences between field and lab subjects.

${ }^{5}$ For a possible explanation of these conflicting findings using subjects from the field in Colombia, see Velez et al. (2010). Running experiments in 3 different locations in Colombia, the authors find that communities that live in collectively owned territories with strong government and non-government presence are more likely to have efficient outcomes when external regulations are imposed while communities that have distant regulatory authorities, nonexistent non-government organizations, and a local fishermen's association are more likely to have efficient outcomes when non-binding verbal agreements are allowed. In a related study, Velez et al. (2012) find that, contrary to theoretical predictions, individual fishermen do not prefer stricter enforcement of fishery regulations.
} 
between our experimental design and theirs. Cardenas et al. (2000) have subjects play with no regulation and then play with weak regulation. Extraction is significantly higher in the last three periods of the weak regulation stage, leading to the conclusion that "external control crowded out other-regarding behavior in favor of greater self-interest" (p. 1730). But there are two important caveats to this conclusion. First, subjects extract more in the last three periods of CPR experiments regardless of the treatment (see Ostrom et al. (1992) for clear evidence). Would extraction have still been higher without the weak regulations? To control for this effect, we run a treatment where the first stage has no regulation and the second stage also has no regulation. Second, it may not be the external control that is causing the problem but the control itself. What if the control was endogenously chosen? To address this issue, we compare weak regulations to the exact same weak regulations. The only difference is that, in one treatment, weak regulations are endogenously chosen and, in the other, the same regulations are exogenously imposed. In the next section, we explain these and other features of our design in detail.

\section{Experimental Design}

To provide a theoretical foundation for our experimental design, we now outline a CPR model under two cases - a baseline case without regulation and a treatment case with monitoring and punishment. Our model largely follows those found in Ostrom et al. (1994), McCarthy et al. (2001), Casari and Plott (2003), and Dorj (2012).

\subsection{Baseline: A Simple Common Pool Resource Model}

Our common pool resource model assumes a fixed number of users, $n$, who have access to the resource. Each user has a fixed endowment of resources $e$ that can be invested either in the common pool resource ("extraction") or in an outside activity. The outside activity 
earns a fixed wage, $w$, while extracting from the common pool resource earns an individual payoff that is dependent on the aggregate group extraction as well as on the user's share in total extraction. Hence we have:

$$
\operatorname{PROFIT}_{i}=w\left(e-x_{i}\right)+\frac{x_{i}}{\sum x_{i}} F\left(\sum x_{i}\right),
$$

where $x_{i}$ is the amount of individual extraction from the common pool resource by individual $i, x_{i} / \sum x_{i}$ is user $i$ 's share in the group extraction, and $F\left(\sum x_{i}\right)$ is the production function that gives the group's return for extracting the common pool resource. $F$ is assumed to be a concave function with $F(0)=0, F^{\prime}(0)>w$ and $F^{\prime}(n e)<0$. Extraction of the common pool resource initially earns higher returns than extraction in an outside activity. However, very large extraction, $x_{l}$, becomes counterproductive, $F^{\prime}\left(x_{l}\right)<0$.

As is standard in the CPR literature, we assume that the profit function is a quadratic function:

$$
F\left(\sum x_{i}\right)=\alpha \sum x_{i}-\beta\left(\sum x_{i}\right)^{2},
$$

where the first term on the right hand side can be thought of as the return from extracting the common pool resource and the second term as the negative externality. Thus, each resource allocated to the common pool confers a private benefit but also imposes a private and a social cost. If users ignore the social cost, too many resources will be extracted relative to the socially optimal amount.

In order to generate predictions regarding behavior in our experiment, we assume that the group of common pool resource users are homogeneous, risk-neutral profit-maximizers with a fixed wage of 0 from the outside activity. Thus, a user's profit function is simply:

$$
\operatorname{PROFIT}_{i}=\alpha x_{i}-x_{i}(\beta X),
$$


where $x_{i}$ can be thought of as user $i$ 's extraction; $\alpha x_{i}$ as the marginal benefit from extraction; and $\beta X$ is the unit cost of extraction, which is a function of the sum of the extraction of all users, where $X=\sum x_{i}$. The strategy set of all users is composed of the set of all real numbers between 0 and $e$ and in each time period, each user decides how much to extract.

The symmetric Nash Equilibrium of this game is for each individual to extract $x_{i}^{*}=$ $(1 /(1+n))(\alpha / \beta)$, which implies a community extraction of $X^{*}=(n /(1+n))(\alpha / \beta) .{ }^{6}$ Because individuals do not fully internalize the negative externality caused by their individual extraction, this decentralized total extraction $X^{*}$ is greater than the total extraction under a benevolent social planner, $X^{S P}=\alpha / 2 \beta$. Thus, the individual extraction under a social planner $x_{i}^{S P}=(1 / n)(\alpha / 2 \beta)$ is less than $x_{i}^{*}$ for all $n \geq 2$.

Numerically, if we let $e=10, \alpha=15, \beta=0.25$, and $n=3$ (as will be the case in our experimental design), we will have:

$$
\operatorname{PROFIT}_{i}=15 x_{i}-x_{i}(0.25 X)
$$

yielding $x_{i}^{*}=15, X^{*}=45, X^{S P}=30, x_{i}^{S P}=10$. Moreover, extraction at the symmetric Nash equilibrium will yield an individual profit of $\operatorname{PROFIT}\left(x_{i}^{*}, x_{-i}^{*}\right)=56.25$ while extraction at the social optimal will yield an individual profit of $\operatorname{PROFIT}\left(x_{i}^{S P}, x_{-i}^{S P}\right)=75$.

\subsection{Treatment: A CPR Model with Monitoring and Punishment}

Since the profit from $x_{i}^{S P}$ is greater than the profit from $x_{i}^{*}$, it is possible for individuals to earn higher profits if they can cooperate with one another. The payoff to cooperation is simply the difference in profits between the social planner optimum and the symmetric Nash equilibrium:

$$
I_{i}^{C}=\operatorname{PROFIT} T_{i}\left(x_{i}^{S P}, x_{-i}^{S P}\right)-\operatorname{PROFIT}\left(x_{i}^{*}, x_{-i}^{*}\right) .
$$

\footnotetext{
${ }^{6}$ It is important to note that, in our setup, asymmetric equilibria exist and lead to different aggregate extraction levels.
} 
However, if an individual believes that others will cooperate and extract what is socially optimal, that individual's best response is to not cooperate. The payoff to not cooperating is simply the difference in profits received when an individual optimally cheats and the profits received when the individual extracts what is socially optimal:

$$
I_{i}^{N C}=\operatorname{PROFIT} T_{i}\left(x_{i}^{N C}\left(x_{-i}^{S P}\right), x_{-i}^{S P}\right)-\operatorname{PROFIT}\left(x_{i}^{S P}, x_{-i}^{S P}\right),
$$

where $x_{i}^{N C}\left(x_{-i}^{S P}\right)=\frac{\alpha-3 \beta x_{-i}^{S P}}{2 \beta}$.

Suppose we wish to force individuals to extract at the socially optimal level. A regulator could announce a punishment for any individual observed deviating from the social optimum. Or the individuals themselves could choose to self-impose a punishment system. Let $s_{i}$ be the fine imposed for being caught cheating. We want to find the fine that will make an individual indifferent between cheating and cooperating when everyone else in the group cooperates, i.e.:

$$
s_{i}=I_{i}^{N C}
$$

Continuing the numerical example from above, this implies that $\operatorname{PROFIT}_{i}\left(x_{i}^{N C}\left(x_{-i}^{S P}\right), x_{-i}^{S P}\right)=$ 100 and $s_{i}=25$. As will be outlined in the next subsection, the expected fine in our experiment can never be large enough to deter a rational risk-neutral agent, since we are primarily interested in studying cooperation in settings where enforcement is weak. In our endogenous treatments, subjects will vote on what the extraction limit should be and on the probability of getting caught if above this limit (monitoring strength). The higher the monitoring strength, the more group members have to pay in enforcement costs. The fine multiplied by the monitoring probability never exceeds 25 , thus there is always an incentive to cheat on the socially optimal limit (if the other members of the group are respecting the limit). 


\subsection{Experiment Procedures}

A total of 12 experiment sessions were conducted on the campus of a US university. There were 153 participants, recruited using the ORSEE software (Greiner 2004). Average earnings were about $\$ 15$ and $\$ 10$ net of the show-up fee paid, and sessions ran for 60 minutes on average. All subjects participated in the same common pool resource game for 10 periods (the Baseline game outlined in Section 3.1) before we introduced an intervention and our different treatments (typically the Treatment game outlined in Section 3.2). We call the first 10 periods "Stage 1" and the 10 periods after the intervention "Stage 2". The experiments were conducted using z-Tree (Fischbacher 2007) and each participant was assigned a computer station with partitions blocking their view of all other stations. Participants were not allowed to verbally communicate with one another for the duration of the experiment. We ran one unpaid pilot with graduate student volunteers and report results from all paid sessions.

Once everyone consented to participating in the experiment, participants were randomly and anonymously placed in groups of three. They remained in the same group for the duration of the experiment and the identities of the other group members were never revealed. Instructions were read out loud and participants were asked to answer a few questions to test their understanding of the instructions. A copy of the full experiment instructions is provided in the Appendix, and a summary of the experimental design is presented in Table 1.

Table 1: Summary of Experimental Details

\begin{tabular}{|l|l|}
\hline Subjects & Undergraduate and Graduate Students \\
\hline Game & Common Pool Resource Game \\
\hline No. of Periods (Stage 1) & 10 periods \\
\hline No. of Periods (Stage 2) & 10 periods \\
\hline No. of Participants/Group & 3 participants in a group \\
\hline Symmetric Nash Equilibrium & An extraction of 15 tokens per person \\
\hline Socially Optimal Solution & An extraction of 10 tokens per person \\
\hline
\end{tabular}

In Stage 1, participants played the baseline CPR game described earlier where they must 
decide how many "tokens" to extract. ${ }^{7}$ They could extract any number between 0 and 20, up to one decimal place. Tokens extracted in one period did not carry over to the next. Once all the participants had entered an extraction amount, each participant was shown her benefit, cost, profit, and the average extraction of the other people in her group.

There are three advantages to making participants play the Stage 1 game in the first 10 periods. First, playing the Stage 1 game ensures proper understanding of the game, especially for participants who have no prior experience playing a CPR game. This guarantees that differences in extraction results between the endogenous and exogenous treatments are due to treatment differences and not to differences in individual participants' knowledge of the game. Second, the desire to govern the commons by imposing management rules is often the result of a perceived tragedy in the rule-less commons. Playing the Stage 1 game therefore makes this historical manifestation more salient. Finally, playing the Stage 1 game before any of the treatments allows us to compare subjects across treatments. This further ensures that differences between treatments are due to treatment effects and not due to differences between subjects.

After these 10 initial periods, we introduced our various interventions by handing out new instructions. We implement 6 treatments in a between-subjects design - No Regulations with No Communication allowed (NoRegWithNC), Endogenous Regulations with No Communication allowed (EndoWithNC), Exogenously Imposed Regulations from the EndoWithNC treatment with No Communication allowed (ExoFromNCWithNC), Endogenous Regulations with Communication (EndoWithC), Exogenously Imposed Regulations from the EndoWithC treatment with Communication allowed between group members (ExoFromCWithC), and Exogenously Imposed Regulations from EndoWithC with No Communication allowed between group members (ExoFromCWithNC). ${ }^{8}$ Subjects in the exogenous regulations treatments were not told where the regulations came from, they were simply introduced as a change in the experiment. Table 2 provides a summary of all the treatments, where the regulations originated, and whether rule-making and communication were allowed. If the endogeneity or the exogeneity of a rule affects individual extraction behavior, we should observe differences between our endogenous and exogenous treatments. If weak regulations truly crowd out cooperative behavior among individuals relative to no regulation whatso-

\footnotetext{
${ }^{7}$ We used the term tokens instead of fish, forests, oil, water, etc. to avoid framing effects.

${ }^{8}$ As a naming convention, we use "Endo" to mean endogenous regulations, "Exo" to mean exogenous regulations, "FromNC" if the regulations are taken from the endogenous with no communication treatment, "FromC" if the regulations are from the endogenous with communication treatment, "WithNC" if communication is not allowed in that treatment, and "WithC" if communication is allowed in that treatment.
} 
ever, we should observe higher extraction in our exogenous treatments compared to Stage 2 of our no regulation, no communication treatment (NoRegWithNC).

Table 2: Treatments Implemented and Description

\begin{tabular}{r|ccc}
\hline \multicolumn{4}{c}{ No Communication Treatments } \\
\hline Treatment Name & NoRegWithNC & EndoWithNC & ExoFromNCWithNC \\
Regulations & none & EndoWithNC & EndoWithNC \\
Self-chosen Rules & no & yes & no \\
Communication & none & no & no \\
\hline \multicolumn{5}{c}{ Communication-Related Treatments } \\
Treatment Name & EndoWithC & ExoFromCWithC & ExoFromCWithNC \\
Regulations & EndoWithC & EndoWithC & EndoWithC \\
Com-chosen Rules & yes & no & no \\
\hline
\end{tabular}

Four of our six treatments can be classified in the standard 2x2 design shown in Table 3: treatments differ by whether the participants created the rules (endogenous) or if the rules were imposed on them (exogenous) and by whether communication within the group was permitted. To analyze the effect of the exogeneity of a rule on individual extraction behavior, we compare participant behavior between the EndoWithNC and ExoFromNCWithNC treatments and between the EndoWithC and ExoFromCWithC treatments. To analyze the effect of communication, we cannot compare across the communication dimension in our $2 \times 2$ treatment because not only is communication changing but so are the caps and monitoring probabilities chosen. We therefore created a final treatment: ExoFromCWithNC. This is a treatment that does not allow for communication but exogenously imposes caps and monitoring probabilities from the endogenous with communication treatment. We can then compare this treatment to ExoFromCWithC to examine whether individual extraction behavior differs in the presence and absence of communication (because now the caps and probabilities are identical across the two treatments). Along with the no regulation, no communication treatment (which tests for the presence of strategic learning and unraveling confounds), this completes the 6 treatments in our experimental design.

Except for NoRegWithNC, participants have individual extraction caps and monitoring probabilities in Stage 2 of all treatments: they either choose these collectively within their group (endogenous treatments) or these are externally imposed on them (exogenous treatments). The exact process for choosing individual caps and monitoring probabilities is explained in the next subsection. Participants were allowed to extract tokens above the cap 
Table 3: 2x2 Experimental Design

\section{Communication \\ No Yes

\begin{tabular}{|c|c|c|c|}
\hline Rule-making & Endogenous & EndoWithNC & EndoWithC \\
\hline & Exogenous & ExoFromNCWithNC & ExoFromCWithC \\
\hline
\end{tabular}

at their own risk. A participant who was monitored and found to have extracted above the cap would have $25 \%$ of their profits for that period confiscated. ${ }^{9}$

Regulation was not costless and monitoring costs increased more than proportionally to the increase in monitoring probability. A $0 \%$ monitoring probability cost 0 , a $10 \%$ monitoring probability cost 1 , a $20 \%$ monitoring probability cost 2.5 , a $30 \%$ monitoring probability cost 4.5, a $40 \%$ monitoring probability cost 7 and a 50\% monitoring probability cost 10 (all in experimental dollars). We did not allow for monitoring probabilities higher than $50 \%$ as we are fundamentally concerned with whether individuals comply with weakly enforced rules. This replicates many field CPRs (especially in the developing world) and most laboratory CPR experiments (Velez et al. 2010). As a reference point, Cardenas et al. (2000) imposed a monitoring probability of $6.25 \%$. Hence, the payoff function for each participant who is monitored and found to have extracted above the cap is:

$$
P A Y O F F=0.75[15 x-x(0.25 X)]-\text { MONITORING COST. }
$$

A participant who extracted at the cap or below it would receive a payoff of:

$$
P A Y O F F=15 x-x(0.25 X)-M O N I T O R I N G C O S T .
$$

\subsection{The Endogenous Treatments}

As mentioned earlier, of the six treatments that we implemented, two can be categorized as belonging to the general endogenous treatments group. These are the EndoWithNC and the EndoWithC treatments. Below, we discuss these two treatments and how they were implemented.

\footnotetext{
${ }^{9} 25 \%$ of $\operatorname{PROFIT}\left(x_{i}^{N C}\left(x_{-i}^{S P}\right), x_{-i}^{S P}\right)=100$ is 25 , the value of the optimal fine with perfect enforcement.
} 


\subsubsection{EndoWithNC Treatment}

After the 10 baseline periods (Stage 1), participants were informed that they would be playing the same common pool resource game with two changes: an extraction cap and a monitoring probability. First, at the start of each period, each participant was asked to vote for the lowest individual extraction cap that she was willing to impose on herself and her group members. Caps could go as low as 0 and as high as 20, and only integer values were allowed. The median vote was then implemented for that period, and the participants were informed what the winning cap was right after voting. Next, each participant had to vote for the highest monitoring probability that she was willing to impose on herself and her group members. Instead of entering integer values, participants were asked to select from a list of possible monitoring probabilities. Higher probabilities involved a higher monitoring cost, which would be deducted from all group members. As with the cap, the median vote was implemented for that period. Participants were asked to vote for both the cap and monitoring probability in every one of the next 10 periods. They were informed of the chosen cap and the chosen monitoring probability before they were asked to play the same common pool resource game as before. At the end of each period, each participant was shown the average extraction of her group members, whether she had been monitored or not, and her profit after monitoring costs and any penalties for exceeding the cap had been deducted (25\% of profits for that period).

\subsubsection{EndoWithC Treatment}

The EndoWithC treatment was identical to the EndoWithNC treatment but with one additional change. Before participants voted for the extraction cap and the monitoring probability in each period, they were allowed to chat with their group members for 2 minutes. Chats were conducted by typing and sending messages through the z-Tree software. Anonymity was preserved as chats were monitored to discourage individuals from revealing their identity, from threatening one another, and from making side payments after the experiment. Once 2 minutes had passed, participants voted for an extraction cap and a monitoring probability before proceeding to the extraction stage. At the end of each period, each participant was shown the same information that the participants in the EndoWithNC treatment were shown. 


\subsection{The Exogenous Treatments}

We took the chosen cap and monitoring probability combinations from the EndoWithNC and EndoWithC treatments and imposed them on the participants in the ExoFromNCWithNC, ExoFromCWithC and ExoFromCWithNC treatments. For example, EndoWithNC treatment caps and monitoring probabilities in one session were all imposed as caps and monitoring probabilities in an ExoFromNCWithNC treatment session (with the exact same number

of groups and in the same chronological order). Likewise, EndoWithC treatment caps and monitoring probabilities in one session were all imposed as caps and monitoring probabilities in an ExoFromCWithC and in an ExoFromCWithNC treatment session. At the end of each period, each participant was shown the same information that participants in the EndoWithNC and EndoWithC treatments were shown.

\subsection{The No Communicaton, No Regulation Treatment}

Unlike the 5 treatments that we have discussed so far, the NoRegWithNC treatment repeats Stage 1 in Stage 2, where there was neither communication among group members nor regulations. After having individuals play the standard common pool resource game for 10 periods, the experimenter interrupted the experiment and told the participants that they will be playing the exact same game with the exact same group for another 10 periods. Between reading the new instructions and the start of Stage 2, the experimenter paused for 1 minute (this meant that all treatments had the same amount of "restart" time between Stage 1 and Stage 2). At the end of each period, participants were shown their extraction, their profit, and the average extraction of their group members.

We ran a total of twelve sessions, two for each treatment. Table 4 shows a summary of the sessions. Note that one group in Session 5 was not matched with any group in Session 7 , since there were 3 less subjects in this session. We include this unmatched group in our analysis unless explicitly mentioned otherwise.

\section{Results}

This section is divided into four main parts. First, we provide a descriptive overview of all treatments. We then investigate differences within each treatment (i.e., Stage 1 vs. Stage 2) before comparing across treatments. We conclude by studying individual choices within a group and exploring how different factors affect an individual's extraction choice. We use 
Table 4: Summary of Sessions

\begin{tabular}{ccccc}
\hline Session & Name & $\begin{array}{c}\text { Exogenous Regulations } \\
\text { from Session }\end{array}$ & Subjects & Groups \\
\hline 1 & EndoWithNC & - & 15 & 5 Groups \\
2 & ExoFromNCWithNC & 1 & 15 & 5 Groups \\
3 & EndoWithC & - & 15 & 5 Groups \\
4 & ExoFromCWithNC & 3 & 15 & 5 Groups \\
5 & EndoWithNC & - & 12 & 4 Groups \\
6 & EndoWithC & - & 9 & 3 Groups \\
7 & ExoFromNCWithNC & 5 & 9 & 3 Groups \\
8 & ExoFromCWithNC & 6 & 9 & 3 Groups \\
9 & ExoFromCWithC & 3 & 15 & 5 Groups \\
10 & ExoFromCWithC & 6 & 9 & 3 Groups \\
11 & NoRegWithNC & - & 15 & 5 Groups \\
12 & NoRegWithNC & - & 15 & 5 Groups \\
\hline
\end{tabular}

the equilibrium predictions from Section 3 as benchmarks and compare them with the actual results from the experiment. It is important to note that we use both non-parametric tests and regression analysis to test our hypotheses and obtain estimates of statistical significance. When we run a non-parametric test, we will nearly always adopt the following approach. We will average the outcome of interest across all relevant periods and within a group. Thus, we do not treat individual observations as independent. An independent observation is the average behavior of a group averaged across all periods. The motivation is straightforward: individual group members may influence each other and the results of earlier periods may influence behavior in later periods. To test for statistically significant differences, we will always report $p$-values for the Wilcoxon-Mann-Whitney (WMW) two-tailed test, unless stated otherwise. Harrison (2007) outlines some of the concerns with using non-parametric tests instead of regression analysis when examining experimental data, especially when key results depend on the absence of statistical significance. For these and other reasons, we supplement all non-parametric tests with random effects regressions of individual behavior, specifically accounting for the panel nature and error structure of the data collected. Unless stated otherwise, all regressions include individual random effects and standard errors are clustered at the group level. 
Figure 1: Mean Extraction and Profit for All Treatments

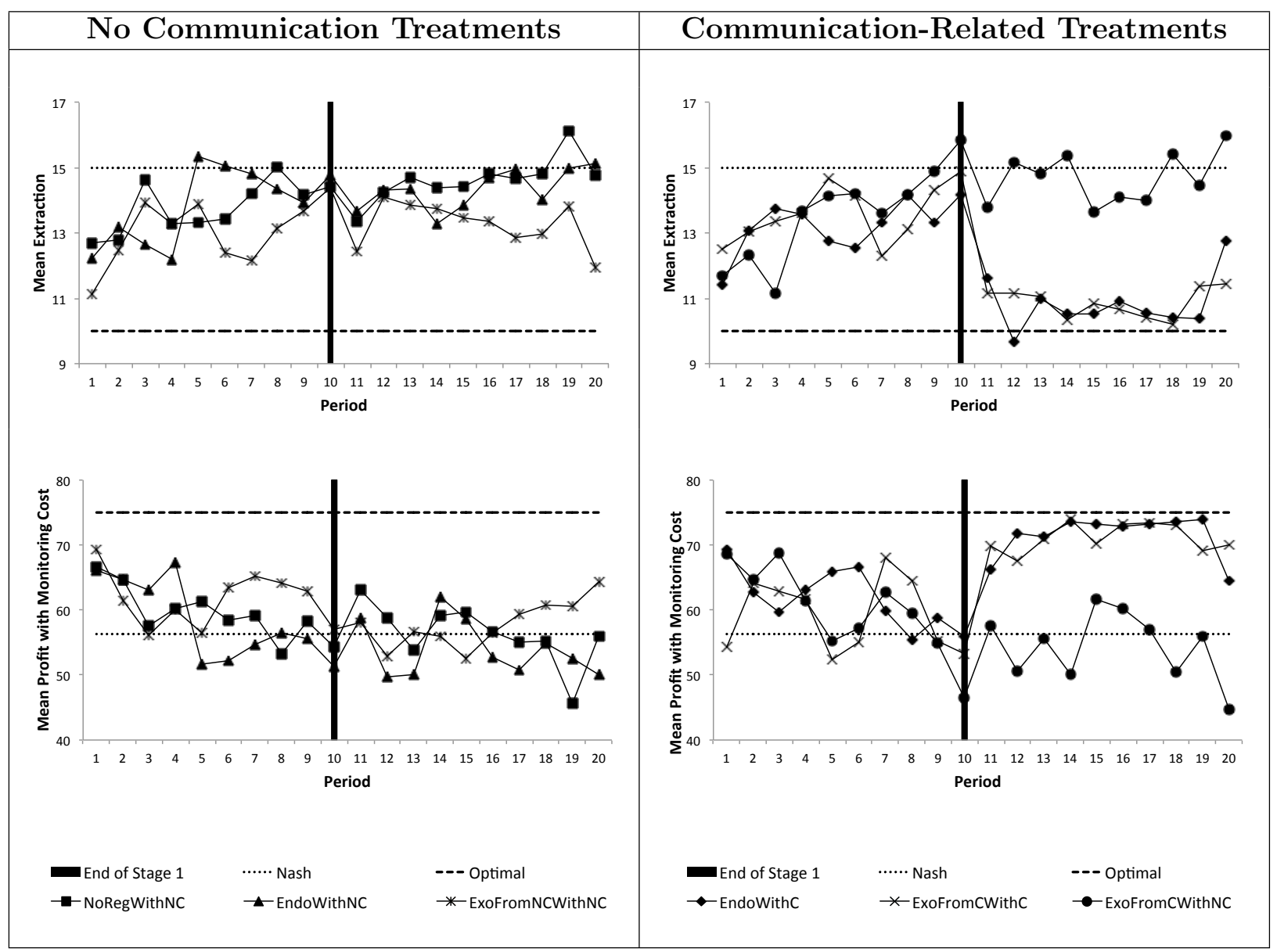

\subsection{Descriptive Overview}

The results of the treatments are shown in Figure 1. The figure shows the mean extraction and profit in every period for each of our 6 treatments. In Stage 1, we observe that subjects extract an average of 13.53 tokens leading to an efficiency of $79.97 \%$, which is well below the social optimum of $100 \%$ efficiency, but above the symmetric Nash prediction of $75 \%$ efficiency. The lowest average extraction happened in the first period with an average extraction of 11.13 tokens while the highest average extraction happened in the last period with an average extraction of 15.34 tokens. These findings are consistent with previous experimental research on common pool resource games and suggest that social norms play an important role for extraction but that strategic learning is taking place over time.

In Stage 2, average extraction is pretty similar for the four treatments with no communication (ranging from 13.26 to 14.68). Conversely, the two treatments that allow for 
communication have much lower average extraction in Stage 2 (10.84 in EndoWithC and 10.87 in ExoFromCWithC). Profits with and without taking monitoring costs in to account do not vary much for the no communication treatments (53 to 56 computer dollars) but are much higher for the communication treatments (71 to 72 computer dollars). While we designed the monitoring costs in such a way that profits should not decrease much after monitoring costs were subtracted, we were surprised by the low monitoring costs chosen. Low monitoring costs imply low monitoring probabilities (an average of $7.29 \%$ ). This suggests that although participants were able to set the cap correctly (the average cap was 11.08 tokens and the optimal cap is 10), over-extraction might still persist due to a very low probability of being monitored.

\subsection{Within Each Treatment (Stage 1 vs. Stage 2)}

Table 5 summarizes average extraction in Stage 1 vs. Stage 2 for all six treatments. As a point of reference, the Nash equilibrium is 15 tokens and the socially optimal extraction level is 10 . Starting with the NoRegWithNC treatment, we observe that the average resource extraction of 14.63 tokens in Stage 2 is greater than average resource extraction of 13.80 tokens in Stage 1. This difference is statistically significantly different from zero $(p=0.0283)$. A random effects regression also shows that Stage 2 extraction is statistically significantly greater than Stage 1 extraction by 0.8287 tokens (see Table 6 ). This result is a clear indication of a strategic learning effect (Ostrom et al. 1992; Chen and Gazzale 2004). When a CPR game is played with repetition, individuals tend to extract more as they learn to play the game better and as they learn how the other members of their group make extraction decisions. This suggests that a CPR experimental design that compares Stage 1 extraction with Stage 2 extraction will tend to be biased towards finding higher extraction levels in Stage 2, as has been the case in the existing literature. Another interesting feature of NoRegWithNC is that we observe separate increasing resource extraction trends in Stage 1 and Stage 2 (see Figure 1). In Period 1, average extraction is 12.7, this rises over time to 14.4 in the 10th period, then drops back down to 13.37 at the start of Stage 2 before rising again to 14.77 in the 20th period. This result suggests a restart effect (Andreoni 1995; Isaac and Walker 1988; Cookson 2000; Croson et al. 2005), however, the difference between the last period of Stage 1 and the first period of Stage 2 is statistically insignificant ( $p=0.2069)$.

Although we observe an increase in resource extraction from Stage 1 to Stage 2 for both the EndoWithNC and ExoFromNCWithNC treatments (see Table 5), translating to a decrease in profits, none of these differences are statistically significantly different from zero. 
Table 5: Two-Tailed Wilcoxon-Mann-Whitney Test: Stage 1 vs. Stage 2 Extraction

\begin{tabular}{lccc}
\hline & NoRegWithNC & $\begin{array}{c}\text { EndoWithNC } \\
(1)\end{array}$ & $\begin{array}{c}\text { ExoFromNCWithNC } \\
(3)\end{array}$ \\
\hline Stage 1: & 13.80 & 13.85 & 13.05 \\
& $(0.24)$ & $(0.38)$ & $(0.65)$ \\
Stage 2: & 14.63 & 14.34 & 13.26 \\
& $(0.24)$ & $(0.43)$ & $(0.64)$ \\
Difference: & 0.83 & 0.48 & 0.21 \\
p-value: & 0.0177 & 0.3320 & 0.3605 \\
\hline & EndoWithC & ExoFromCWithC & ExoFromCWithNC \\
& $(4)$ & $(5)$ & $(6)$ \\
\hline Stage 1: & 13.21 & 13.60 & 13.58 \\
& $(0.64)$ & $(0.79)$ & $(0.38)$ \\
Stage 2: & 10.84 & 10.87 & 14.68 \\
& $(0.37)$ & $(0.30)$ & 1.10 \\
Difference: & -2.37 & -2.73 & 0.0524 \\
p-value: & 0.0524 & 0.0141 & \\
\hline
\end{tabular}

Notes: Standard errors in parentheses.

The insignificance of the non-parametric tests is confirmed in random effects regressions in the top half of Table 6. Furthermore, resource extraction before and after the treatment intervention under the EndoWithNC and ExoFromNCWithNC treatments are very similar, in both absolute and relative terms. On average, extraction increased by $3.53 \%$ in Stage 2 in the EndoWithNC treatment, and by $1.61 \%$ in the ExoFromNCWithNC treatment. Profits declined by $5.37 \%$ in Stage 2 in the EndoWithNC treatment and by $4.20 \%$ in the ExoFromNCWithNC treatment. Thus, holding everything else constant, the exogenous or endogenous nature of the rule-making does not appear to affect extraction or payoffs. In the next section, we test whether these small differences between the two treatments are statistically significant.

We do observe statistically significant differences between Stage 1 and Stage 2 average extraction for all of our communication-related treatments. EndoWithC and ExoFromCWithC allow for communication while ExoFromCWithNC doesn't allow communication but uses the caps and monitoring probabilities chosen by the endogenous regulation treatment that allowed for communication. We find the most significant difference between Stage 1 and Stage 2 in our EndoWithC and ExoFromCWithC treatments, with average resource extraction 
Table 6: Stage 1 vs. Stage 2 Random Effects Regressions Dependent Variable: Extraction of Tokens

\begin{tabular}{|c|c|c|c|}
\hline & $\begin{array}{c}\text { NoRegWithNC } \\
(1)\end{array}$ & $\begin{array}{c}\text { EndoWithNC } \\
\text { (2) }\end{array}$ & $\begin{array}{c}\text { ExoFromNCWithNC } \\
(3)\end{array}$ \\
\hline \multirow[t]{2}{*}{ Stage 2} & $0.8287^{* * *}$ & 0.5458 & 0.2129 \\
\hline & $(0.3057)$ & $(0.3778)$ & $(0.3655)$ \\
\hline \multirow[t]{2}{*}{ Constant } & $13.8027^{* * *}$ & $13.8333^{* * *}$ & $13.0500 * * *$ \\
\hline & $(0.3384)$ & $(0.5693)$ & $(0.6339)$ \\
\hline \multirow[t]{2}{*}{$\mathrm{N}$} & 600 & 480 & 480 \\
\hline & $\begin{array}{l}\text { EndoWithC } \\
(4)\end{array}$ & $\begin{array}{c}\text { ExoFromCWithC } \\
(5)\end{array}$ & $\begin{array}{c}\text { ExoFromCWithNC } \\
(6)\end{array}$ \\
\hline \multirow{2}{*}{ Stage 2} & $-2.3737 * * *$ & $-2.7283^{* * *}$ & $1.1029 * * *$ \\
\hline & $(0.291)$ & $(0.2949)$ & $(0.3446)$ \\
\hline \multirow[t]{2}{*}{ Constant } & $13.2112^{* * *}$ & $13.5975^{* * *}$ & $13.5779 * * *$ \\
\hline & $(0.3715)$ & $(0.4231)$ & $(0.545)$ \\
\hline $\mathrm{N}$ & 480 & 480 & 480 \\
\hline
\end{tabular}

Notes: The variable Stage 2 is a dummy variable that takes on the value of 0 if the participants are in Stage 1 and a value of 1 if the participants are in Stage 2. Robust standard errors clustered at the group level in parentheses.

*** $p<0.01,{ }^{* *} p<0.05,{ }^{*} p<0.10$ 
decreasing by $17.96 \%$ for the EndoWithC treatment and $20.07 \%$ for the ExoFromCWithC treatment. This translates to an increase in profits without monitoring costs of $16.59 \%$ for the EndoWithC treatment and $21.76 \%$ for the ExoFromCWithC treatment. This difference in resource extraction is statistically different from zero (EndoWithC $p=0.0046$, ExoFromCWithC $p=0.0380$ ). There is also a significant difference between the Stage 1 and Stage 2 average resource extraction in the ExoFromCWithNC treatment but in the opposite direction. Average resource extraction increased by $8.10 \%(p=0.0830)$. The bottom half of Table 6 confirms these statistical differences using random effects regressions.

Extractions in Stage 2 for the EndoWithC and ExoFromCWithC treatments are closer to the social optimum and hence, more efficient, while extractions in Stage 2 for the ExoFromCWithNC treatment are higher than the average resource extraction for the baseline periods and hence, less efficient. Efficiency under the EndoWithC treatment is $95.24 \%$ and 95.6\% under ExoFromCWithC, while efficiency under the ExoFromCWithNC treatment is only $72.49 \%$. This result demonstrates that not all exogenously imposed rules lead to less efficiency. ExoFromCWithC and ExoFromCWithNC have the exact same rules imposed exogenously but completely different outcomes. The difference is driven by the presence of communication. Comparing EndoWithC with ExoFromCWithNC and then concluding that the difference is due to exogenous regulations crowding out intrinsic motivations would be misleading.

\subsection{Across Treatments}

If it is assumed that subjects are not different across treatments, then a test for the effect of the treatment interventions can simply compare behavior in Stage 2. Wilcoxon-MannWhitney tests fail to reject the null hypothesis that the resource extraction in Stage 1 is the same across any two treatments. ${ }^{10}$ The regression results in Table 7 Column (1) confirm this finding. Hence, there are no statistically significant differences across groups in the different treatments and a comparison of just the treatment periods is justified.

\footnotetext{
${ }^{10} p$ : NoRegWithNC vs. EndoWithNC, 1.000; NoRegWithNC vs. ExoFromNCWithNC, 0.4598; NoRegWithNC vs. EndoWithC, 0.4598; NoRegWithNC vs. ExoFromCWithC, 0.8286; NoRegWithNC vs. ExoFromCWithNC, 0.5726; EndoWithNC vs. ExoFromNCWithNC, 0.3704; EndoWithNC vs. EndoWithC, 0.3704; EndoWithNC vs. ExoFromCWithC, 0.8784; EndoWithNC vs. ExoFromCWithNC, 0.4808; ExoFromNCWithNC vs. EndoWithC, 0.7984; ExoFromNCWithNC vs. ExoFromCWithC, 0.4418; ExoFromNCWithNC vs. ExoFromCWithNC, 0.5738; EndoWithC vs. ExoFromCWithC, 0.7210; EndoWithC vs. ExoFromCWithNC, 0.6454; ExoFromCWithC vs. ExoFromCWithNC, 0.4418.
} 


\section{Table 7: Random Effects Regressions Across Treatments}

Dependent Variable: Extraction of Tokens

\begin{tabular}{|c|c|c|c|c|c|c|}
\hline & \multirow{2}{*}{$\begin{array}{l}\text { Stage } 1 \\
\text { All } \\
\text { (1) }\end{array}$} & \multirow[b]{2}{*}{$\begin{array}{l}\text { All } \\
\text { (2) }\end{array}$} & \multicolumn{3}{|c|}{ Stage 2} & \multirow[b]{2}{*}{$\begin{array}{c}\text { Comm vs. No Comm } \\
\text { (Exo) } \\
(6)\end{array}$} \\
\hline & & & $\begin{array}{l}\text { Endo vs. Exo } \\
\text { (No Comm) } \\
(3)\end{array}$ & $\begin{array}{l}\text { Endo vs. Exo } \\
\text { (Comm) } \\
(4)\end{array}$ & $\begin{array}{c}\text { Comm vs. No Comm } \\
\text { (Endo) } \\
(5)\end{array}$ & \\
\hline EndoWithNC & $\begin{array}{c}0.0514 \\
(0.4263)\end{array}$ & $\begin{array}{l}-0.2943 \\
(0.4742)\end{array}$ & & & & \\
\hline ExoFromNCWithNC & $\begin{array}{l}-0.7527 \\
(0.6576)\end{array}$ & $\begin{array}{c}-1.3684^{* *} \\
(0.6468)\end{array}$ & & & & \\
\hline EndoWithC & $\begin{array}{l}-0.5914 \\
(0.6447)\end{array}$ & $\begin{array}{c}-3.7938^{* * * *} \\
(0.423)\end{array}$ & & & & \\
\hline ExoFromCWithC & $\begin{array}{l}-0.2052 \\
(0.7799)\end{array}$ & $\begin{array}{c}-3.7622^{* * *} \\
(0.3675)\end{array}$ & & & & \\
\hline ExoFromCWithNC & $\begin{array}{l}-0.2248 \\
(0.4291)\end{array}$ & $\begin{array}{c}0.0495 \\
(0.5117)\end{array}$ & & & & \\
\hline Exogenous & & & $\begin{array}{c}1.0741 \\
(0.7453)\end{array}$ & $\begin{array}{l}-0.0317 \\
(0.4624)\end{array}$ & & \\
\hline Communication & & & & & $\begin{array}{c}-3.4995 * * * \\
(0.5535)\end{array}$ & $\begin{array}{c}-3.8117^{* * *} \\
(0.5481)\end{array}$ \\
\hline Constant & $\begin{array}{c}13.8027^{* * *} \\
(0.2317)\end{array}$ & $\begin{array}{c}14.6313^{* * *} \\
(0.2338)\end{array}$ & $\begin{array}{c}13.2629^{* * *} \\
(0.6151)\end{array}$ & $\begin{array}{c}10.8692^{* * *} \\
(0.2898)\end{array}$ & $\begin{array}{c}14.3370^{* * *} \\
(0.4208)\end{array}$ & $\begin{array}{c}14.6808^{* * *} \\
(0.4652)\end{array}$ \\
\hline $\mathrm{N}$ & 1530 & 1530 & 510 & 480 & 510 & 480 \\
\hline
\end{tabular}

Notes: The treatment names are dummy variables that take on the value of 1 if an observation belongs to the treatment and 0 otherwise. We have a total of 6 treatments, NoRegWithNC, EndoWithNC, ExoFromNCWithNC, EndoWithC, ExoFromCWithC, and ExoFromCWithNC. Column (1) runs a random effects regression using all observations in Stage 1 while Column (2) runs the same regression using all observations in Stage 2. NoRegWithNC is the omitted dummy variable. Column (3) uses all observations in Stage 2 of the EndoWithNC and ExoFromNCWithNC treatments, where the variable Exogenous is a dummy variable which takes on a value of 1 if the treatment is exogenous and 0 if the treatment is endogenous. Column (4) uses all observation in Stage 2 of the EndoWithC and ExoFromCWithC treatments, where the variable Exogenous is a dummy variable which takes on a value of 1 if the treatment is exogenous and 0 if the treatment is endogenous. Column (5) uses all observations in Stage 2 of the EndoWithC and EndoWithNC treatments, where the variable Communication takes on the value of 1 if the treatment allowed for communication and 0 otherwise. Column (6) uses all observations in Stage 2 of the ExoFromCWithC and ExoFromCwithNC treatments, where the variable Communication takes on the value of 1 if the treatment allowed for communication and 0 otherwise. Robust standard errors clustered at the group level in parentheses.

*** $p<0.01,{ }^{* *} p<0.05,{ }^{*} p<0.10$ 
Cardenas et al. (2000) concluded that external regulations crowd out the intrinsic motivation of individuals to cooperate by comparing Stage 1 with Stage 2 in their exogenous regulation treatment. They found that extraction increased from Stage 1 to Stage 2 (but only if they restricted their attention to the last three periods in each stage). We find a similar increase in extraction behavior in our two exogenous regulations without communication treatments. However, we know from our NoRegWithNC treatment that a statistically significant difference in extraction between Stage 1 and Stage 2 can occur even when no regulations are imposed. We refer to this temporal trend as the strategic learning effect. Hence, if the increase in extraction in Stage 2 for ExoFromNCWithNC and ExoFromCWithNC is due to external regulation crowding out other-regarding behavior, we should observe a clear difference between these two treatments and our NoRegWithNC treatment. We do not find this result ( $p$ : NoRegWithNC vs. ExoFromNCWithNC, 0.1458; NoRegWithNC vs. ExoFromCWithNC, 0.7518). The regression results in Column (2) of Table 7 confirm this finding and demonstrate that the exogeneity of a rule-making process does not affect extraction behavior. In fact, the coefficient on ExoFromNCWithNC indicates that exogenously imposed but weakly enforced regulations outperform having no regulations. Instead of simply failing to reject a null of no difference, we clearly reject a null of exogenous regulations being worse than no regulations. This is the opposite of the main conclusion in Cardenas et al. (2000). Thus, we do not find any statistical support for the hypothesis that regulations crowd out cooperation and present our first main result.

Result 1. (Crowding Out Effect) Weak external regulations do not crowd out intrinsic motivations to reduce extraction. Our two exogenous regulations without communication treatments do not have statistically significantly higher extraction compared to our no regulation treatment.

Even if exogenous regulations don't crowd out intrinsic motivations, it could be the case that endogenous regulations crowd in cooperative behavior. Thus, endogenously chosen regulations would be preferable. If we hypothesize that individuals who are involved in the rule-making are more likely to follow the rules they've created, we should find resource extraction in Stage 2 in the endogenous treatments (EndoWithNC and EndoWithC) to be less than resource extraction in the exogenous treatments (ExoFromNCWithNC and ExoFromCWithC). ${ }^{11}$ Comparing average resource extraction between these two treatments, we find that the above hypothesis is not supported. Average token extraction in the EndoWithNC

\footnotetext{
${ }^{11}$ It is very important to note that we are deriving this hypothesis from claims made in the existing literature. There are other potential mechanisms that could lead to higher compliance and/or lower extraction.
} 
treatment (14.34) is not statistically significantly different from average token extraction (13.26) in the ExoFromNCWithNC treatment $(p=0.3704)$. A 10.84 average token extraction in the EndoWithC treatment is also not statistically significantly different from a 10.87 average token extraction in the ExoFromCWithC treatment $(p=0.721)$. When we conduct a further robustness check - subtracting the Stage 1 averages from the Stage 2 averages and comparing these - the same result holds ( $p$ : EndoWithNC vs. ExoFromNCWithNC, 0.7430; EndoWithC vs. ExoFromCWithC, 0.7210). Regression tests including subject level random effects (Columns (3) and (4) of Table 7) confirm this second main result.

Result 2. (Endogenous vs. Exogenous Effect) The endogeneity or exogeneity of a rule does not affect extraction behavior in our experiment. We find no statistically significant differences in resource extraction between the EndoWithNC and ExoFrom NCWithNC treatments and between the EndoWithC and ExoFromCWithC treatments.

We now examine the causal impact of communication. When the same caps and monitoring probabilities are implemented in two separate groups, where one group was allowed to communicate while the other was not, we find extreme differences in individual resource extraction behavior. From Figure 1 and Table 5, we see that average extraction in Stage 2 under the ExoFromCWithC treatment (10.87 tokens) was significantly lower than the average extraction under the ExoFromCWithNC treatment (14.58 tokens) $(p=0.0002)$. This result is confirmed in a random effects regression in Column (6) of Table 7. Hence, under the exact same regulations, communication encourages individuals to extract less and move closer to the social optimum. Column (5) of Table 7 compares communication with no communication but in both treatments the regulations were endogenously chosen. The communication effect is similar in magnitude but this regression is not an "apples-to-apples" comparison since the chosen regulations are not identical across treatments. These findings motivate our third main result.

Result 3. (Communication Effect) Individuals who were allowed to communicate with their group members extracted less. Given the same caps and monitoring probabilities, extraction under the ExoFromCWithC treatment is significantly less than extraction under the ExoFromCWithNC treatment.

\subsection{Individual Behavior}

We now analyze individual voting and extraction behavior, in particular: (1) if individuals are able to select the correct cap, (2) how the chosen cap affects votes for monitoring 
Table 8: Mean Caps and Monitoring Probabilities for the Endogenous Treatments

\begin{tabular}{lccc}
\hline & Mean EndoWithNC & Mean EndoWithC & $\boldsymbol{p}$-value \\
\hline Voted Cap & 11.31 & 10.48 & 0.5414 \\
& $(0.96)$ & $(0.46)$ & \\
Voted Monitoring (\%) & 14.85 & 5.54 & $0.0112^{* *}$ \\
Winning Cap & $(1.97)$ & $(2.10)$ & \\
& 11.79 & 10.30 & 0.4234 \\
Winning Monitoring (\%) & $(1.19)$ & $(0.58)$ & \\
& 9.22 & 5.13 & 0.2766 \\
\hline
\end{tabular}

Notes: Standard errors in parentheses. $p$-values are from WMW tests.

Figure 2: Winning Caps and Monitoring Probabilities

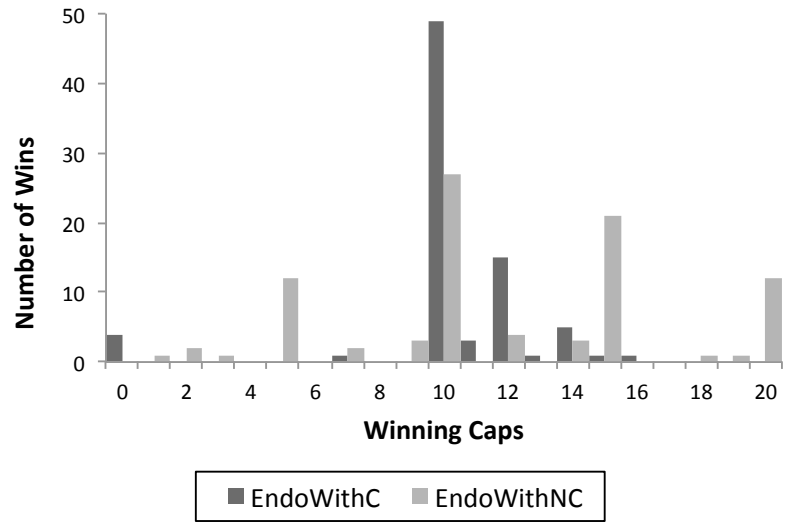

(a) Frequency of Winning Caps

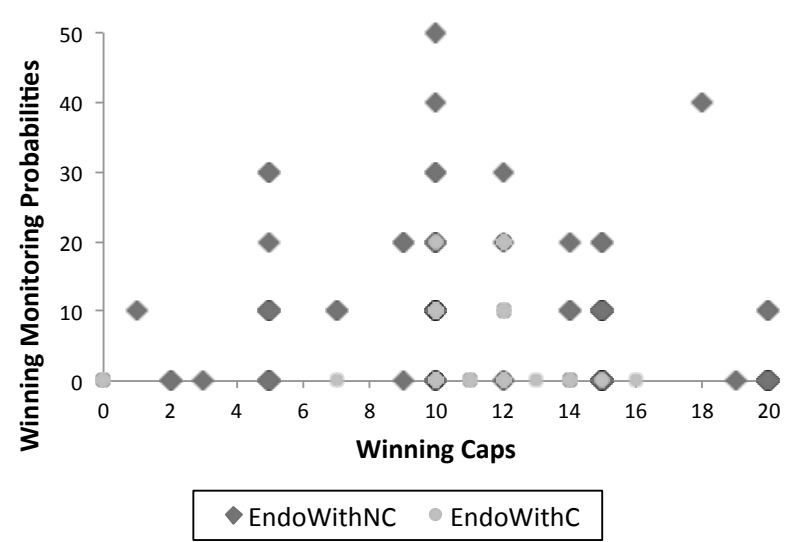

(b) Monitoring Probabilities by Caps

probabilities, and (3) how the chosen cap and monitoring probability influence extraction decisions. We also explore how communication (or the lack of it) affects individual votes for caps and monitoring probabilities, which, in turn, affect resource extraction behavior. Table 8 presents the mean of the winning caps and monitoring probabilities for our two endogenous treatments and Figure 2 visualizes the relationship between the winning caps and monitoring probabilities. We find that, on average, groups are able to get very close to the optimal cap in both the EndoWithNC and EndoWithC treatments (the socially optimal cap is 10). The mean cap for both groups is not statistically different from 10 at the $5 \%$ level of significance. Subjects clearly understand the game and how to solve the social dilemma. We also find that individuals in the communication treatment tend to vote for lower monitoring 
probabilities on average than individuals in the no communication treatment. The regression results in Table 9 confirm that there are no statistical differences in cap votes and chosen caps across the two treatments. We do observe significant differences in the monitoring probabilities. Votes in the EndoWithC treatment were on average 9 percentage points lower and the chosen probability was 5 percentage points lower (Columns (3) and (4) of Table 9. Our interpretation of this result is the following. It is well documented in laboratory experiments that communication reduces social distance and builds trust. If subjects trust each other, there is no need to invest in costly monitoring and enforcement, raising profits for all group members. Thus, it is very likely that many groups in the communication treatment decided it was unnecessary to pay for enforcement.

Table 9: Regression Results: Effect of Communication on Regulation

\begin{tabular}{|c|c|c|c|c|c|}
\hline Dependent Variable: & $\begin{array}{c}\text { Cap Votes } \\
(1)\end{array}$ & $\begin{array}{c}\text { Chosen Cap } \\
(2)\end{array}$ & $\begin{array}{c}\text { MP Votes } \\
(3)\end{array}$ & $\begin{array}{c}\text { Chosen MP } \\
(4)\end{array}$ & $\begin{array}{c}\text { MP Votes } \\
(5)\end{array}$ \\
\hline Communication & $\begin{array}{l}-0.9292 \\
(1.1429)\end{array}$ & $\begin{array}{c}-1.85 \\
(1.3635)\end{array}$ & $\begin{array}{c}-9.2917^{* * *} \\
(2.967)\end{array}$ & $\begin{array}{c}-5.25 \\
(3.3889)\end{array}$ & \\
\hline Equal or Stricter & & & & & $\begin{array}{c}-2.0313^{* * *} \\
(0.6792)\end{array}$ \\
\hline Constant & $\begin{array}{c}11.4125^{* * *} \\
(1.0514)\end{array}$ & $\begin{array}{c}12.1500^{* * *} \\
(1.2415)\end{array}$ & $\begin{array}{c}14.8333^{* * *} \\
(2.1591)\end{array}$ & $\begin{array}{c}10.3750 * * * \\
(2.4155)\end{array}$ & $\begin{array}{c}5.6348 \text { *** } \\
(2.1331)\end{array}$ \\
\hline $\mathrm{N}$ & 480 & 160 & 480 & 160 & 240 \\
\hline
\end{tabular}

Notes: All regressions were done using observations from the endogenous treatments (EndoWith $\mathrm{C}$ and EndoWithNC). The variable Communication is a dummy variable that takes on the value of 1 if communication is allowed (EndoWithC) and 0 otherwise. The dummy variable Equal or Stricter is 1 if the chosen cap is equal to or stricter than the cap a subject voted for. MP stands for Monitoring Probability. Robust standard errors clustered at the group level in parentheses.

*** $p<0.01,{ }^{* *} p<0.05,{ }^{*} p<0.10$

We now explore item (2): how the chosen cap affects votes for monitoring probabilities. Figure 3 presents the frequency distributions of votes for monitoring probabilities conditional on being in one of three categories: (1) the winning cap is lower than the cap the individual voted for, (2) the winning cap is higher than the cap the individual voted for, and (3) the winning cap is equal to the cap the individual voted for. In the EndoWithNC treatment, we do not observe striking visual differences in the voting for probabilities, conditional on being outvoted or winning the vote. In percentage terms, the distributions of votes are almost identical. The main interesting pattern we observe is for the EndoWithC treatment. When 
an individual votes for a cap that is lower than the one chosen, there is a clear desire to increase enforcement since the majority of votes are for $20 \%$ monitoring. When the cap is equal to the one desired (or more stringent), subjects vote for low monitoring probabilities. The statistical significance of this pattern is confirmed in Column (5) of Table 9 where we see that votes for monitoring probabilities are 2 percentage points lower if the cap is equal to or stricter than the one desired. Our interpretation of this result is that observing a cap in line with what a subject would like to see implemented serves as a signal that other members of the group have similar preferences. Thus, there is less of a need for monitoring and punishment. Note that in EndoWithC, the winning cap equals a subject's vote $89 \%$ of the time so there are very few instances where subjects are out-voted.

Table 10: Logit Regressions: Compliance

\begin{tabular}{|c|c|c|c|c|}
\hline Treatments: & $\begin{array}{l}\text { All } \\
(1)\end{array}$ & $\begin{array}{c}\text { EndoWith } \mathrm{C} \\
(2)\end{array}$ & $\begin{array}{c}\text { Endogenous } \\
(3)\end{array}$ & $\begin{array}{c}\text { Exogenous } \\
(4)\end{array}$ \\
\hline Communication & $\begin{array}{c}1.1884^{* * *} \\
(0.4153)\end{array}$ & & & \\
\hline Stricter Cap & & $\begin{array}{c}-1.4115^{* * *} \\
(0.5411)\end{array}$ & & \\
\hline $\mathrm{MP} \geq 40 \%$ & & & $\begin{array}{l}-0.1761 \\
(0.7046)\end{array}$ & $\begin{array}{c}1.7067^{* * *} \\
(0.6094)\end{array}$ \\
\hline Constant & $\begin{array}{c}0.5961^{* * *} \\
(0.2181)\end{array}$ & $\begin{array}{c}1.9711^{* * *} \\
(0.5176)\end{array}$ & $\begin{array}{c}0.8693^{* * *} \\
(0.2801)\end{array}$ & $\begin{array}{c}0.3728 \\
(0.2463)\end{array}$ \\
\hline $\mathrm{N}$ & 1530 & 240 & 510 & 720 \\
\hline
\end{tabular}

Notes: Dependent variable is compliance. All regressions are logit regressions. Robust standard errors clustered at the group level in parentheses. *** $p<0.01,{ }^{* *} p<0.05,{ }^{*} p<0.10$

Combining all of our treatments, we find that individuals complied with the winning cap more than $60 \%$ of the time (788 out of 1230 instances). Sixty-one percent of the 36 individuals who were caught exceeding the cap exceeded the cap again in the next period. This suggests that the majority of the subjects who exceeded were not affected by being monitored. The treatments that allowed communication among group members have the highest compliance rates (caps are respected $87 \%$ of the time under the EndoWithC treatment and $85 \%$ of the time under the ExoFromCWithC treatment) while the ExoFromCWithNC treatment has the lowest frequency of compliance (caps are respected only $30 \%$ of the time). The statistical significance of this difference in compliance rates is confirmed in a logit regression in Column 
Figure 3: Votes for Monitoring Probabilities Conditional on Votes for Caps

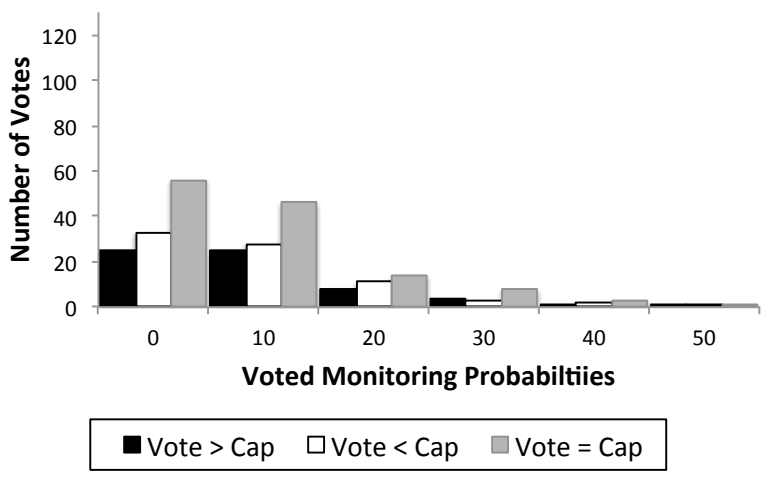

(a) EndoWithNC Treatment

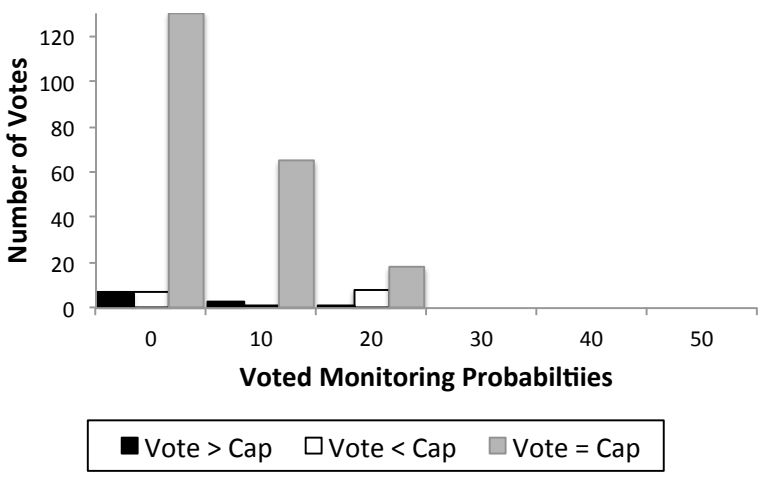

(b) EndoWithC Treatment

(1) Table 10.

We now explore whether individuals exceeded their caps, conditional on whether the winning cap is lower, higher or equal to the cap they voted for. Figure 4 shows the percentage of individuals who exceeded the caps separated by treatment and by whether the winning cap is greater than, equal to, or less than the cap the individual voted for. Under the EndoWithNC treatment, individuals are most likely to exceed the cap when it was greater than the cap that they voted for. However, this difference is not statistically significant. When individuals are allowed to communicate with one another, none of the subjects exceeded the cap when it was greater than what they voted for, while $36 \%$ exceeded the cap when it was less than what they vote for. A regression in Column (2) of Table 10 confirms the statistical significance of this difference in compliance rates. One plausible interpretation is that these are subjects who don't want to reduce extraction and don't want low caps. We find that many who didn't adhere to the winning cap when it was what they voted for seemed to do so for two reasons - (1) being in one particular group that adopted a bizarre strategy or (2) an experiment that was about to end. Out of the 28 instances of disobedience, 15 were done by individuals belonging to Group 1 in Session 3. Over 50\% of these violations were due to this group voting for and implementing a cap of zero tokens. This group attempted to take turns having one individual extract 20 and everyone else extract 0 , but it never worked out. As for the remaining 13 cases of disobedience, 6 of them occurred in the last period of the experiment.

We now turn our attention to compliance, conditional on enforcement. Figure 5 reveals 
Figure 4: \% of Individuals Who Exceeded Winning Caps Conditional on Vote

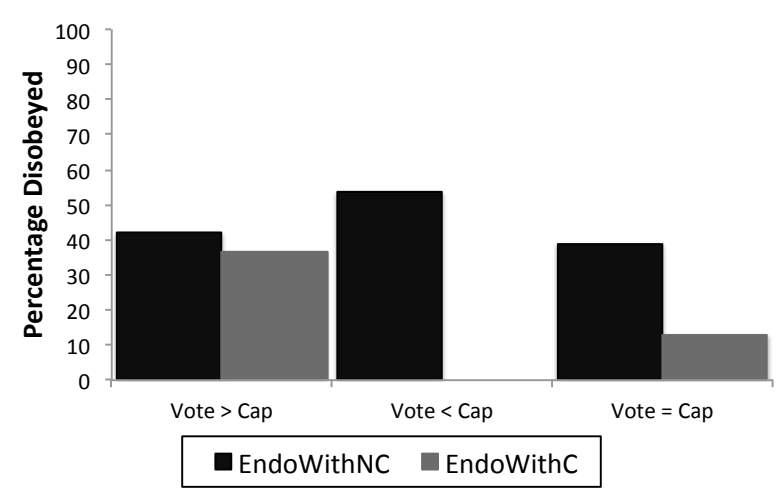

that as monitoring probabilities increase for the ExoFromNCWithNC, ExoFromCWithC and ExoFromCWithNC treatments, so does the percentage of individuals who comply with the cap. However, this monotonic relationship does not hold for endogenously chosen probabilities and actually goes in the opposite direction. The statistical significance of this divergence is confirmed in Columns (3) and (4) of Table 10, which presents logit regressions of monitoring probability on compliance for the endogenous and exogenous treatments, respectively. It appears that the standard deterrent hypothesis holds when rules are exogenously imposed but not when they are endogenously chosen. This is a different result to Tyran and Feld (2006) and worthy of further investigation. We do not have a satisfactory explanation for why the deterrent hypothesis does not hold under the endogenous treatments. But this stark difference in behavior is certainly suggestive of a real difference in behavior when rules are endogenously chosen instead of exogenously imposed.

\section{Conclusion}

This paper presents results from an experiment designed to: (1) analyze whether the endogeneity or exogeneity of a rule affects an individual's intrinsic motivation to reduce extraction in a CPR game, and (2) disentangle the confounding effects of strategic learning and communication from the effect of exogenous regulations. To test our hypotheses, we construct six treatments that differ in terms of: (1) whether communication was permitted among group members, (2) whether regulations existed, and (3) whether regulations were chosen by the group or exogenously imposed by a third party. When neither communication nor 
Figure 5: \% of Individuals Who Complied with the Cap

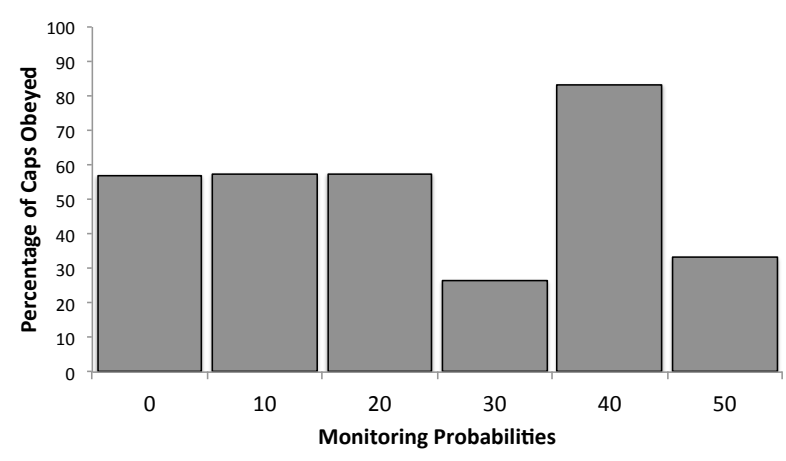

(a) EndoWithNC Treatment

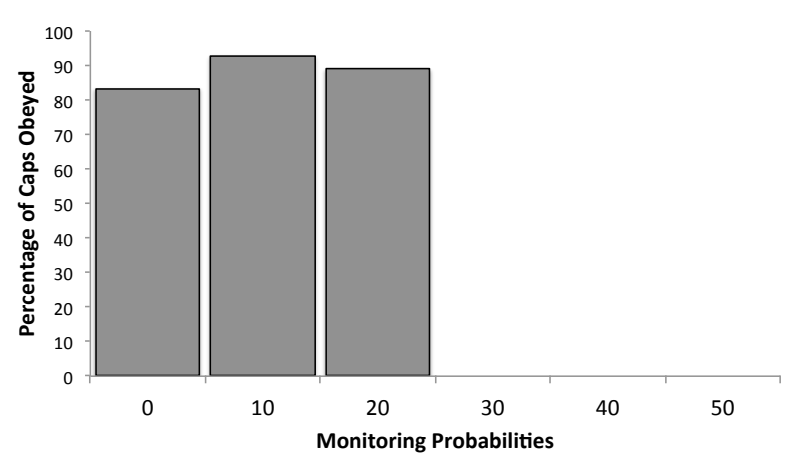

(c) EndoWithC Treatment

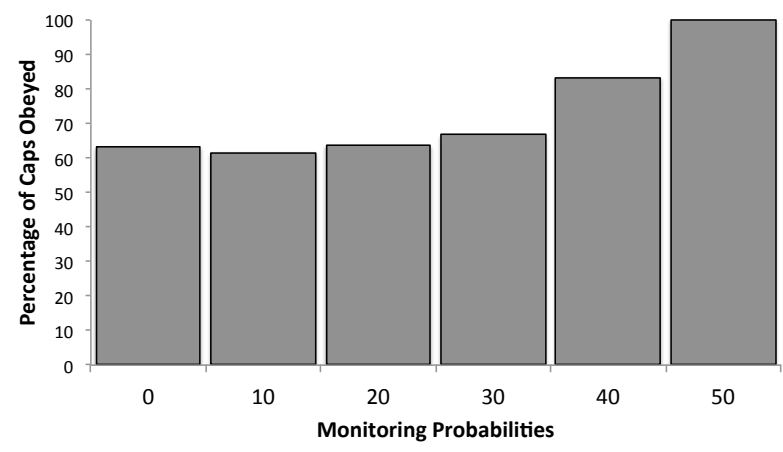

(b) ExoFromNCWithNC Treatment

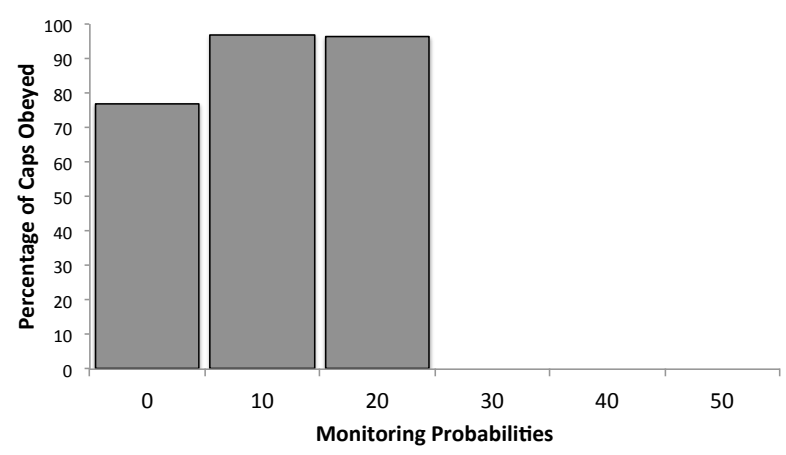

(d) ExoFromCWithC Treatment

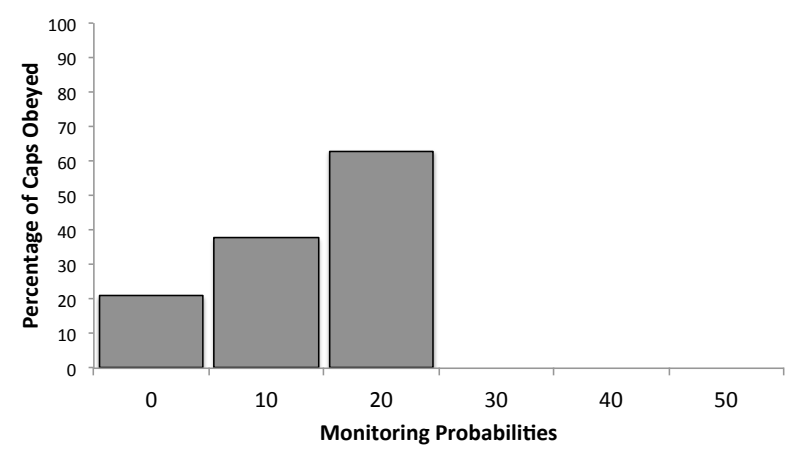

(e) ExoFromCWithNC Treatment 
regulations are allowed, we find an increasing trend in individual extraction across periods. By comparing this treatment to our exogenous treatments that do not allow communication, we find that extraction under our externally imposed regulations treatments is never higher than extraction under the no regulation treatment. In one treatment, exogenously imposed but weakly enforced regulations lead to statistically significantly less extraction than having no regulations. This strongly suggests that externally imposed regulations do not crowd out cooperation among common pool resource users in this type of experiment.

Our results also demonstrate that resource extraction behavior does not differ significantly when rules are endogenously created versus exogenously imposed. In a key departure from earlier work, our exogenous regulations are exactly the same as the endogenously chosen regulations. We also confirm a well-established result that communication leads to lower resource extraction. Compliance with the rules is also higher when communication is permitted compared to when only voting is permitted. When the caps and monitoring probabilities from the EndoWithC treatment are imposed exogenously in a treatment that does not allow communication, extraction rises and profits fall. But when these same caps are exogenously imposed and subjects are allowed to communicate, we observe no differences. This stresses the importance of controlling for communication effects and other potential confounds in these types of laboratory experiments.

Overall, we find no evidence that external regulation crowds out the intrinsic motivation of individuals to reduce extraction. We also find that how the rules are chosen makes no difference in whether individuals choose to comply with the rules. Communication and strategic learning matter, but these are separate effects to regulation crowding out effects. We believe it is unfair to claim that there is strong evidence to support the crowding out hypothesis in CPR experiments. 


\section{References}

Anderson, J., Burks, S., Carpenter, J., Götte, L., Maurer, K., Nosenzo, D., Potter, R., Rocha, K., and Rustichini, A. (2013). Self-selection and variations in the laboratory measurement of other-regarding preferences across subject pools: evidence from one college student and two adult samples. Experimental Economics, 16(2):170-189.

Andreoni, J. (1995). Cooperation in public-goods experiments: kindness or confusion? The American Economic Review, pages 891-904.

Bischoff, I. (2007). Institutional choice versus communication in social dilemmas: an experimental approach. Journal of Economic Behavior \& Organization, 62(1):20 - 36.

Cardenas, J.-C. (2004). Norms from outside and from inside: an experimental analysis on the governance of local ecosystems. Forest Policy and Economics, 6:229-241. Economics of Sustainable Forest Management.

Cardenas, J.-C., Stranlund, J., and Willis, C. (2000). Local environmental control and institutional crowding-out. World Development, 28(10):1719-1733.

Carpenter, J. and Myers, C. K. (2010). Why volunteer? Evidence on the role of altruism, image, and incentives. Journal of Public Economics, 94:911-920.

Casari, M. and Plott, C. (2003). Decentralized management of common property resources: experiments with a centuries-old institution. Journal of Economic Behavior \& Organization, 51(2):217-247.

Charness, G. (2000). Responsibility and effort in an experimental labor market. Journal of Economic Behavior \& Organization, 42(3):375-384.

Chen, Y. and Gazzale, R. (2004). When does learning in games generate convergence to Nash equilibria? The role of supermodularity in an experimental setting. The American Economic Review, 9(5):1505-1535.

Cookson, R. (2000). Framing effects in public goods experiments. Experimental Economics, $3(1): 55-79$.

Croson, R., Fatas, E., and Neugebauer, T. (2005). Reciprocity, matching and conditional cooperation in two public goods games. Economics Letters, 87(1):95-101. 
Deci, E., Koestner, R., and Ryan, R. (1999). A meta-analytic review of experiments examining the effects of extrinsic rewards on intrinsic motivation. Psychological Bulletin, $125(6): 627$.

Dorj, D. (2012). Common-pool resources with free mobility. Economic Inquiry, 51(2):14041420.

Fischbacher, U. (2007). z-tree: Zurich toolbox for ready-made economic experiments. Experimental Economics, 10:171-178.

Frey, B. and Oberholzer-Gee, F. (1997). The cost of price incentives: An empirical analysis of motivation crowding-out. The American Economic Review, 87(4):746-755.

Gneezy, U. and Rustichini, A. (2000a). A fine is a price. Journal of Legal Studies, 29:1.

Gneezy, U. and Rustichini, A. (2000b). Pay enough or don't pay at all. The Quarterly Journal of Economics, 115(3):791-810.

Greiner, B. (2004). An online recruitment system of economic experiments. In Kurt Kremer, V. M., editor, Forschung und wissenschaftliches Rechnen, volume 63, pages 79-93. GWDG Bericht 63, Ges. Fr Wiss. Datenverarbeitung: Gttingen.

Harrison, G. W. (2007). House money effects in public good experiments: Comment. Experimental Economics, 10:429-437.

Isaac, R. and Walker, J. (1988). Communication and free-riding behavior: The voluntary contribution mechanism. Economic Inquiry, 26(4):585-608.

Kunreuther, H. and Easterling, D. (1990). Are risk-benefit tradeoffs possible in siting hazardous facilities? The American Economic Review, 80(2):252-256.

McCarthy, N., Sadoulet, E., and de Janvry, A. (2001). Common pool resource appropriation under costly cooperation. Journal of Environmental Economics and Management, $42(3): 297-309$.

Ostrom, E. (1990). Governing the Commons: The Evolution of Institutions for Collective Action. Cambridge University Press.

Ostrom, E. (1992). Crafting institutions for self-governing irrigation systems. ICS Press San Francisco. 
Ostrom, E. (2000). Collective action and the evolution of social norms. The Journal of Economic Perspectives, 14(3):137-158.

Ostrom, E. (2009). Nobel prize lecture.

Ostrom, E., Gardner, R., and Walker, J. (1994). Rules, games, and common-pool resources. University of Michigan Press.

Ostrom, E., Walker, J., and Gardner, R. (1992). Covenants with and without a sword: Self-governance is possible. The American Political Science Review, 86(2):404-417.

Titmuss, R. M. (1970). The Gift Reltionship. Allen and Unwin London.

Tyran, J.-R. and Feld, L. P. (2006). Achieving compliance when legal sanctions are nondeterrent. Scandinavian Journal of Economics, 108(1):135-156.

Velez, M. A., Murphy, J. J., and Stranlund, J. K. (2010). Centralized and decentralized management of local common pool resources in the developing world: Experimental evidence from fishing communities in colombia. Economic Inquiry, 48(2):254-265.

Velez, M. A., Stranlund, J. K., and Murphy, J. J. (2012). Preferences for government enforcement of a common pool harvest quota: Theory and experimental evidence from fishing communities in Colombia. Ecological Economics, 77(0):185-192.

Vyrastekova, J. and Soest, D. v. (2003). Centralized common-pool management and local community participation. Land Economics, 79(4):500-514. 


\section{Appendices}

\section{A Baseline Experiment Instructions \\ EXPERIMENT INSTRUCTIONS}

\section{Introduction}

Welcome to my experiment. For showing up on time, I will pay you a $\$ 5$ show-up fee. In addition, you may receive additional earnings as the result of the outcomes in the experimental session. Today's session will take about an hour. Please do not communicate with other participants during the experiment.

\section{Details}

At the beginning of this decision making experiment you will be matched with two other people, randomly selected from the people in this room, to form a group of three. You will remain in this group of three people for the duration of the whole decision-making experiment. The identity of the other members of your group will never be revealed. You are going to make decisions with the other members of your group for 10 periods. Each period, you must decide how many tokens to extract: you can extract any number between 0 and 20. You can extract fractions of tokens, up to one decimal place. Tokens extracted in one period do not carry over to the next.

The tokens that you extract each round will earn you a cash BENEFIT, which I describe below. But, any tokens you extract will also COST you money, which I also describe below.

IMPORTANT NOTICE: You should consider all monetary values (including your profits) as "computer dollars". At the end of the experiment we will pay you in cash an amount equal to $\$ 1.00$ for every 100 computer dollars you earn.

\section{BENEFITS FROM TOKENS YOU EXTRACT}

Let's denote the number of tokens you extract as $x$. The benefit to you from extracting tokens is given by the following formula:

$$
B E N E F I T=15 x
$$

At the end of these instructions is a table labeled TABLE 01: BENEFITS FROM TOKENS. You can use this table during the experiment to inform your decisions. For example, let's say you extract 10 tokens in a given decision period. That token extraction will earn you 
BENEFITS of 150 computer dollars. Study this table carefully. If you have any questions raise your hand and I will help you.

Extracting tokens earns you a cash BENEFIT. But, there is a cost for all the tokens that you extract. This cost depends both on the amount you extract and the amount everyone else in your group extracts.

\section{TOKEN COSTS}

In each period, what you pay for the tokens that you extract equals the number of tokens you extract times the Unit Token Cost for that period. The Unit Token Cost depends on how many tokens you extract, as well as how many tokens the other members of your group extract. Let's denote the total number of tokens extracted by your group (including yourself) as $X$. Then the Unit Token Cost is as follows:

$$
\text { UNIT TOKEN COST }=\frac{1}{4} X=0.25 X
$$

If your group extracted a total of 30 tokens, the Unit Token Cost would be 7.5. Your individual cost for the period equals the number of tokens you extract times the Unit Token Cost:

$$
\text { INDIVIDU AL COST }=x(0.25 X)
$$

Now let's assume you had extracted 10 and the other members of your group had extracted 20. Your individual cost would thus be $10^{*} 7.5=75$ computer dollars. Please look at the tables at the end of these instructions and find the table labeled "TABLE 02: COST FROM TOKENS". This table shows how your individual cost changes as you extract more and more tokens AND the other members of your group extract more and more tokens.

Your profit for each period is equal to the benefit minus the individual cost:

$$
P R O F I T=B E N E F I T-I N D I V I D U A L C O S T=15 x-x(0.25 X)
$$

To continue the example, if you extracted 10 tokens and the other members of your group extracted a total of 20 , then your profit for the period would be $150-75=75$ computer dollars. Please look at the tables at the end of these instructions and find the table labeled TABLE 03: PROFITS FROM TOKENS. This table shows how your profit changes as you extract more and more tokens AND the other members of your group extract more and more tokens.

The total profit earned by your group thus depends on your decisions and the decisions of your group members. If no one in your group extracts any tokens, no one will earn any profit. If your group extracts 30 tokens, the group will earn a total profit of 225 . If your group extracts 45 tokens, the group will earn a total profit of 168.75. If the group extracts 60 tokens, no one will earn any profit. 
Individually, you earn the highest profit when your group members only extract a few tokens and you extract as many tokens as possible. For example, if your group members each extract 10 tokens and you extract 20 tokens, you will earn a profit of 100 .

Each period, you choose how much to extract. At the end of each period, your benefit, cost, and profit will be revealed. The average extraction of the two members of your group will also be revealed.

After the ten periods are over, you will take part in a different decision making game. Once both of these experiments are over, the computer will sum your profit from every period and I will pay you in cash in private. Remember that each computer dollar is worth one cent of real money.

I highly encourage clarifying questions. Thank you for your participation.

\section{REVIEW}

1. How many members are there in a group?

2. Suppose you extracted 5 tokens and the rest of your group extracted 10 tokens. What is:
a. Your benefit?
b. Your cost?
c. Your profit?

\section{TABLE 01: BENEFITS FROM TOKENS}

This Table Displays the Benefits for Various Token Extractions 


\begin{tabular}{|c|c|}
\hline Tokens & Benefits \\
\hline 0 & 0 \\
\hline 1 & 15 \\
\hline 2 & 30 \\
\hline 3 & 45 \\
\hline 4 & 60 \\
\hline 5 & 75 \\
\hline 6 & 90 \\
\hline 7 & 105 \\
\hline 8 & 120 \\
\hline 9 & 135 \\
\hline 10 & 150 \\
\hline 11 & 165 \\
\hline 12 & 180 \\
\hline 13 & 195 \\
\hline 14 & 210 \\
\hline 15 & 225 \\
\hline 16 & 240 \\
\hline 17 & 255 \\
\hline 18 & 270 \\
\hline 19 & 285 \\
\hline 20 & 300 \\
\hline
\end{tabular}




\section{TABLE 02: COST FROM TOKENS}

This Table Displays Costs for Various Individual and Group Token Extractions

\begin{tabular}{|c|c|c|c|c|c|c|}
\hline & \multicolumn{5}{|c|}{$\begin{array}{c}\text { The Number of Tokens } \\
\text { You Extract }\end{array}$} \\
\hline & & 0 & 5 & 10 & 15 & 20 \\
\hline \multirow{9}{*}{ 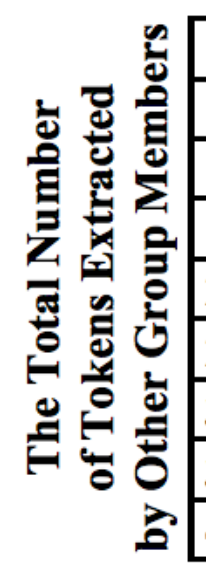 } & 0 & 0 & 6.25 & 25 & 56.25 & 100 \\
\hline & 5 & 0 & 12.5 & . & 75 & 125 \\
\hline & 10 & 0 & 18.75 & 50 & 93.75 & 150 \\
\hline & 15 & 0 & 25 & 2 . & 112 & 175 \\
\hline & 20 & 0 & 31.25 & 75 & 131.25 & 200 \\
\hline & 25 & 0 & 37.5 & 87. & 150 & 225 \\
\hline & 30 & 0 & 43.75 & 00 & 168.75 & 250 \\
\hline & 35 & 0 & 50 & 112.5 & 187.5 & 275 \\
\hline & 40 & 0 & 56.25 & 125 & 206.25 & 300 \\
\hline
\end{tabular}

TABLE 03: PROFIT FROM TOKENS

This Table Displays Profits for Various Individual and Group Token Extractions

\begin{tabular}{|c|c|c|c|c|c|c|}
\hline & \multicolumn{5}{|c|}{$\begin{array}{c}\text { The Number of Tokens } \\
\text { You Extract }\end{array}$} \\
\hline & & 0 & 5 & 10 & 15 & 20 \\
\hline & 0 & 0 & 68.75 & 125 & 168.75 & 200 \\
\hline & 5 & 0 & 62.5 & 112.5 & 150 & 175 \\
\hline & 10 & 0 & 56.25 & 100 & 131.25 & 150 \\
\hline & 15 & 0 & 50 & 87.5 & 112.5 & 125 \\
\hline & 20 & 0 & 43.75 & 75 & 93.75 & 100 \\
\hline & 25 & 0 & 37.5 & 62.5 & 75 & 75 \\
\hline & 30 & 0 & 31.25 & 50 & 56.25 & 50 \\
\hline & 35 & 0 & 25 & 37.5 & 37.5 & 25 \\
\hline & 40 & 0 & 18.75 & 25 & 18.75 & 0 \\
\hline
\end{tabular}




\section{B EndoNC Treatment Experiment Instructions EXPERIMENT INSTRUCTIONS - PART II}

We are now going to run the same decision making experiment with two additional changes.

First, in every period, you will need to vote for the lowest extraction cap that you are willing to impose on yourself and your group members. The median extraction cap will be implemented. For example, if you voted for a cap of 10 and your group members voted for a cap of 12 and 15, a cap of 12 will be implemented.

IMPORTANT NOTICE: You can still extract tokens more than your cap but there is a probability of getting caught and getting fined for extracting more than your cap which I will discuss below.

Second, in every period, you will need to vote for a probability of getting monitored that you are willing to impose on yourself and your group members. The median monitoring probability will be implemented. For example, if you voted for a monitoring probability of $10 \%$ and your members voted for a monitoring probability of $20 \%$ and $30 \%$, a monitoring probability of $20 \%$ will be implemented for that period. Please turn to the table labeled TABLE 04: COST FROM MONITORING to see the corresponding costs for each monitoring probability.

After voting, the experiment will proceed as before.

\section{MONITORING AND FINE}

At the end of each period, the computer will randomly decide to monitor each individual in your group based on the monitoring probability. If you are monitored and then found to be above your cap, $25 \%$ of your profits for that period will be confiscated. Hence, if you extracted above your cap, your payoff will be:

$$
\begin{aligned}
\text { PAYOFF } & =0.75(\text { EXTRACTION PROFIT })-\text { MONITORING COST } \\
& =0.75(B E N E F I T S-C O S T)-\text { MONITORING COST } \\
& =0.75[15 x-x(0.25 X)]-\text { MONITORING COST }
\end{aligned}
$$

Otherwise, it will be:

$$
\begin{aligned}
\text { PAYOFF } & =\text { EXTRACTIONPROFIT - MONITORING COST } \\
& =\text { BENEFIT }- \text { COST }- \text { MONITORING COST } \\
& =15 x-x(0.25 X)-\text { MONTORING COST }
\end{aligned}
$$

For example, suppose an individual cap of 10 tokens and a monitoring probability of $10 \%$ have have been chosen by your group. If you extract 20 tokens and everyone else in your 
group extracts 10 tokens, you will have a profit of 100 from extraction. There is a $10 \%$ chance that you will be monitored, and if you are, your profit will decrease by 25 and your new profit would be 75 . Since your group has to pay for this $10 \%$ monitoring, your profit will also decrease by 1 . Hence, your payoff for this period will either be 74 computer dollars (100 - $25-1)$ if you get monitored or 99 computer dollars $(100-1)$ if you dont.

Everything else is the same as before. Please raise your hand if you have any questions.

\section{REVIEW}

1. If your extraction profit is 100, you get monitored and found to be above your cap, and you paid for a $10 \%$ monitoring probability, what will your payoff be?

2. How much will it cost you individually if there is a monitoring probability of $20 \%$ ?

\section{TABLE 04: COST FROM MONITORING}

This Table Displays the Costs for Various Monitoring Probabilities

\begin{tabular}{|c|c|}
\hline PROBABILITY & COST \\
\hline $0 \%$ & 0 \\
\hline $10 \%$ & 1 \\
\hline $20 \%$ & 2.5 \\
\hline $30 \%$ & 4.5 \\
\hline $40 \%$ & 7 \\
\hline $50 \%$ & 10 \\
\hline
\end{tabular}




\section{EndoC Treatment Experiment Instructions EXPERIMENT INSTRUCTIONS - PART II}

We are now going to run the same decision making experiment with three additional changes.

First, in every period, you and your group members will be given 2 minutes to chat online with one another to decide on your group's cap, enforcement, and extraction strategy for the period.

Second, in every period, you will need to vote for the lowest extraction cap that you are willing to impose on yourself and your group members. The median extraction cap will be implemented. For example, if you voted for a cap of 10 and your group members voted for a cap of 12 and 15, a cap of 12 will be implemented.

IMPORTANT NOTICE: You can still extract tokens more than your cap but there is a probability of getting caught and getting fined for extracting more than your cap which I will discuss below.

Third, in every period, you will need to vote for a probability of getting monitored that you are willing to impose on yourself and your group members. The median monitoring probability will be implemented. For example, if you voted for a monitoring probability of $10 \%$ and your members voted for a monitoring probability of $20 \%$ and $30 \%$, a monitoring probability of $20 \%$ will be implemented for that period. Please turn to the table labeled "TABLE 04: COST FROM MONITORING" to see the corresponding costs for each monitoring probability.

After voting, the experiment will proceed as before.

\section{MONITORING AND FINE}

At the end of each period, the computer will randomly decide to monitor each individual in your group based on the monitoring probability. If you are monitored and then found to be above your cap, $25 \%$ of your profits for that period will be confiscated. Hence, if you extracted above your cap, your payoff will be:

$$
\begin{aligned}
\text { PAYOFF } & =0.75(\text { EXTRACTION PROFIT })-\text { MONITORING COST } \\
& =0.75(\text { BENEFITS }- \text { COST })-\text { MONITORING COST } \\
& =0.75[15 x-x(0.25 X)]-\text { MONITORING COST }
\end{aligned}
$$

Otherwise, it will be:

$$
\begin{aligned}
\text { PAYOFF } & =\text { EXTRACTIONPROFIT - MONITORING COST } \\
& =\text { BENEFIT }- \text { COST }- \text { MONITORING COST } \\
& =15 x-x(0.25 X)-\text { MONTORING COST }
\end{aligned}
$$


For example, suppose an individual cap of 10 tokens and a monitoring probability of $10 \%$ have have been chosen by your group. If you extract 20 tokens and everyone else in your group extracts 10 tokens, you will have a profit of 100 from extraction. There is a $10 \%$ chance that you will be monitored, and if you are, your profit will decrease by 25 and your new profit would be 75 . Since your group has to pay for this $10 \%$ monitoring, your profit will also decrease by 1 . Hence, your payoff for this period will either be 74 computer dollars (100 - $25-1)$ if you get monitored or 99 computer dollars (100 - 1) if you dont.

Everything else is the same as before. Please raise your hand if you have any questions.

\section{REVIEW}

1. If your extraction profit is 100 , you get monitored and found to be above your cap, and you paid for a $10 \%$ monitoring probability, what will your payoff be?

2. How much will it cost you individually if there is a monitoring probability of $20 \%$ ? 
TABLE 04: COST FROM MONITORING

This Table Displays the Costs for Various Monitoring Probabilities

\begin{tabular}{|c|c|}
\hline PROBABILITY & COST \\
\hline $0 \%$ & 0 \\
\hline $10 \%$ & 1 \\
\hline $20 \%$ & 2.5 \\
\hline $30 \%$ & 4.5 \\
\hline $40 \%$ & 7 \\
\hline $50 \%$ & 10 \\
\hline
\end{tabular}




\section{ExoNC and ExoC Treatments Experiment Instruc- tions}

\section{EXPERIMENT INSTRUCTIONS - PART II}

We are now going to run the same decision making experiment with two additional changes.

For every period, a cap and a monitoring probability will be imposed on you and your group members. Every monitoring probability has a corresponding cost and this cost will be subtracted from your profits from extraction. Please turn to the table labeled TABLE 04: COST FROM MONITORING to see the corresponding cost for each monitoring probability.

Other than this, the experiment will proceed as before.

\section{MONITORING AND FINE}

At the end of each period, the computer will randomly decide to monitor each individual in your group based on the monitoring probability. If you are monitored and then found to be above your cap, $25 \%$ of your profits for that period will be confiscated. Hence, if you extracted above your cap, your payoff will be:

$$
\begin{aligned}
\text { PAYOFF } & =0.75(\text { EXTRACTION PROFIT })-\text { MONITORING COST } \\
& =0.75(\text { BENEFITS }- \text { COST })-\text { MONITORING COST } \\
& =0.75[15 x-x(0.25 X)]-\text { MONITORING COST }
\end{aligned}
$$

Otherwise, it will be:

$$
\begin{aligned}
\text { PAYOFF } & =\text { EXTRACTION PROFIT }- \text { MONITORING COST } \\
& =\text { BENEFIT }- \text { COST }- \text { MONITORING COST } \\
& =15 x-x(0.25 X)-\text { MONTORING COST }
\end{aligned}
$$

For example, suppose an individual cap of 10 tokens and a monitoring probability of $10 \%$ have have been chosen by your group. If you extract 20 tokens and everyone else in your group extracts 10 tokens, you will have a profit of 100 from extraction. There is a $10 \%$ chance that you will be monitored, and if you are, your profit will decrease by 25 and your new profit would be 75 . Since your group has to pay for this $10 \%$ monitoring, your profit will also decrease by 1 . Hence, your payoff for this period will either be 74 computer dollars (100 - 25 - 1) if you get monitored or 99 computer dollars (100 - 1) if you dont.

Everything else is the same as before. Please raise your hand if you have any questions.

\section{REVIEW}


1. If your extraction profit is 100 , you get monitored and found to be above your cap, and you paid for a $10 \%$ monitoring probability, what will your payoff be?

2. How much will it cost you individually if there is a monitoring probability of $20 \%$ ?

\section{TABLE 04: COST FROM MONITORING}

This Table Displays the Costs for Various Monitoring Probabilities

\begin{tabular}{|c|c|}
\hline PROBABILITY & COST \\
\hline $0 \%$ & 0 \\
\hline $10 \%$ & 1 \\
\hline $20 \%$ & 2.5 \\
\hline $30 \%$ & 4.5 \\
\hline $40 \%$ & 7 \\
\hline $50 \%$ & 10 \\
\hline
\end{tabular}

